\title{
Verification of Markov hypothesis for conserved scalar process: validation of conditional moment closure for turbulent combustion
}

\author{
Andrew P. Wandel* Nigel S. A. Smith ${ }^{\dagger}$ \\ A. Y. Klimenko
}

(Received 1 October 2001; revised 12 June 2002)

\begin{abstract}
The Conditional Moment Closure (CMC) is a model for turbulent combustion that describes the physics of the process across the flow for a wide range of situations. The theoretical basis used in closing the model is that conserved
\end{abstract}

*Department of Mechanical Engineering, The University of Queensland, Brisbane, Australia. mailto:a.wandel@mailbox.uq.edu.au

${ }^{\dagger}$ Aeronautical and Maritime Research Laboratory, Defence Science and Technology Organisation, Melbourne, Australia.

${ }^{\ddagger}$ Department of Mechanical Engineering, The University of Queensland, Brisbane, Australia.

${ }^{0}$ See http://anziamj.austms.org.au/V44/CTAC2001/Wand for this article, (C) Austral. Mathematical Soc. 2003. Published 1 April 2003. ISSN 1446-8735 
scalars develop in turbulent fields similarly to Markov processes. We describe the formulation of $\mathrm{CMC}$ in both its deterministic and stochastic forms and show some preliminary results justifying the assumption of similarity with the Markov hypothesis.

\section{Contents}

1 Introduction

C803

2 Theory

C804

3 Results

C807

4 Analysis

C812

5 Conclusions

C815

6 Future work

C817

References

C817

\section{Introduction}

The Conditional Moment Closure (CMC) is a model for describing the transport of reactive scalars in conserved scalar spaces for turbulent combustion processes. It was proposed by Klimenko [6], who derived it from the joint PDF equation, then independently by Bilger [1] via decomposition of the reactive scalar into its conditional average and conditional fluctuations (see also [7]). Although unconditional averages are used in modelling the fluid mechanics, this 
is not possible for modelling chemical reactions because properties are highly non-linear. When the first conditional moment closure is considered, it is possible to close the source term; however, this procedure introduces additional terms which must be closed using other means.

CMC has been tested against an array of experimental and numerical results (see [7] for an extensive list), with Kim, Huh and Tao [5] implementing the full CMC. However, while these tests exhibit support for the validity of the model, they do not directly examine the hypothesis that was made to complete the derivation $[6,7]$ : similarity of a conserved scalar process with a Markov process. In this paper we present some preliminary findings which indicate that this is the case hence the only assumption made in the derivation of $\mathrm{CMC}$ is shown to be valid.

\section{Theory}

The deterministic formulation of $\mathrm{CMC}$ is a transport equation for the conditional expectation $Q=\langle Y \mid \xi=Z\rangle$ :

$$
\frac{\partial Q}{\partial t}+\langle\mathbf{v} \mid Z\rangle \cdot \nabla Q-\langle N \mid Z\rangle \frac{\partial^{2} Q}{\partial Z^{2}}=\langle W \mid Z\rangle .
$$

Here $Y$ is a reactive scalar (for combustion purposes mass fraction) conditioned on the random variable $\xi$ being a particular conserved scalar value: the sample space variable $Z$ (the mass fraction of fuel). Also, $\mathbf{v}$ is the physical velocity, $N$ the scalar dissipation rate $\left[N=D(\nabla Z)^{2}, D\right.$ being the diffusivity], $W$ the chemical source term and, for ease of notation, $\langle\bullet \mid Z\rangle \equiv\langle\bullet \mid \xi=Z\rangle$. Coupled with the CMC equation is the PDF equation:

$$
\frac{\partial P}{\partial t}+\nabla \cdot\langle\mathbf{v} \mid Z\rangle P+\frac{\partial^{2}\langle N \mid Z\rangle P}{\partial Z^{2}}=0,
$$


where $P$ is the Eulerian scalar PDF. When solving the system Equations (1)-(2), either the PDF must be assigned a presumed shape or the conditional scalar dissipation rate modelled separately. We define the Lagrangian PDF as:

$$
F \equiv P Q / n,
$$

where $n=$ const so that $\int_{Z} F d Z=1$, and combine Equations (1) and (2) to take the form of the Fokker-Planck (direct Kolmogorov) equation

$$
\frac{\partial F}{\partial t}+\nabla \cdot\langle\mathbf{v} \mid Z\rangle F+\frac{\partial A F}{\partial Z}-\frac{\partial^{2} B F}{\partial Z^{2}}=\frac{P\langle W \mid Z\rangle}{n} .
$$

The drift and diffusion coefficients are, respectively,

$$
A(Z, t) \equiv \frac{2}{P(Z, t)} \frac{\partial\langle N \mid Z\rangle P(Z, t)}{\partial Z} \text { and } B(Z, t) \equiv\langle N \mid Z\rangle .
$$

Equation (4) is equivalent to the Ito stochastic equation

$$
d Z^{*}=A\left(Z^{*}, t\right) d t+\left[2 B\left(Z^{*}, t\right)\right]^{1 / 2} d w
$$

where the superscript $*$ represents a model for the process and $d w$ a Wiener process. We call Equation (6) "the stochastic formulation of CMC" and may use it as a model for CMC instead of Equations (1) or (4). For further details, refer to [8].

For the purposes of this paper, we consider $Y$ to be the concentration of massless particles carried with the flow. The physical velocities of these particles, $\mathbf{v}_{i}$, is taken to be identical to that of the flow at the particles' locations: $\mathbf{v}_{i}=\mathbf{v}\left(\mathbf{x}_{i}(t), t\right)$. The subscript $i$ denotes an individual particle, $i=1, \ldots, n[n$ being the total number of particles, used in Equation (3)]. The value of the conserved scalar of a particle is similarly defined; we define the rate of change 
of passive scalar as the scalar velocity, $u_{i}(t) \equiv d Z_{i} / d t$. The source term is zero.

To test the hypothesis of similarity with a Markov process, we consider the autocorrelation functions for the scalar velocity

$$
\rho_{u}\left(t_{1}, t_{2}\right) \equiv \frac{\left\langle u_{i}^{\prime \prime}\left(t_{1}\right) u_{i}^{\prime \prime}\left(t_{2}\right)\right\rangle}{\left\langle\left[u_{i}^{\prime \prime}\left(t_{1}\right)\right]^{2}\right\rangle},
$$

and physical velocity

$$
\rho_{\mathbf{v}}\left(t_{1}, t_{2}\right) \equiv \frac{\left\langle\mathbf{v}_{i}^{\prime}\left(t_{1}\right) \cdot \mathbf{v}_{i}^{\prime}\left(t_{2}\right)\right\rangle}{\left\langle\mathbf{v}_{i}^{\prime}\left(t_{1}\right) \cdot \mathbf{v}_{i}^{\prime}\left(t_{1}\right)\right\rangle} .
$$

Here $t_{1}<t_{2}$, the prime superscript denotes fluctuations about the unconditional mean $\left(\mathbf{v}_{i}^{\prime}=\mathbf{v}_{i}-\langle\mathbf{v}\rangle\right)$ and double prime superscript fluctuations about the conditional mean $\left(u_{i}^{\prime \prime}=u_{i}-\langle u \mid Z\rangle\right)$. To obtain average values for $\rho_{\mathbf{v}}$ across all three components, the dot product was applied, rather than considering just one component. We note that the correlation of the process described by Equation (6) is proportional to the Dirac delta function, $\rho_{u^{*}}\left(t_{1}, t_{2}\right) \propto \delta\left(t_{2}-t_{1}\right)$. We do not expect $Z_{i}(t)$ to be mathematically identical to $Z_{i}^{*}(t)$, but we do expect it to be closer to a Markov process than the equivalent physical process, $\mathbf{x}_{i}(t)$. So the characteristic time taken for $\rho_{u}$ to become zero is considered to be significantly shorter than the equivalent $\rho_{\mathbf{v}}$. If true, the diffusion equation [Equation (4)] is a much better model than conventional modelling which is based on turbulent diffusion in physical space.

To justify this stance, we consider estimations based on the theory of the inertial interval [6]. When the observation time is of the order of the inertial interval, $u$ can be considered to be stationary since the particle remains in an area of fluid which is locally homogeneous. The two parameters of the inertial interval are the respective dissipation rates in physical and scalar space and the dispersion of 
particles is dependent only on these parameters:

$$
\left\langle\left(\Delta Z_{i}\right)^{2}\right\rangle=\left\langle N \mid Z_{0}\right\rangle \Delta t
$$

Here $\Delta Z_{i} \equiv Z_{i}-Z_{0}$ and $\Delta t \equiv t-t_{0}$ and the correlation function for this process is

$$
\rho_{u}(\Delta t)=\frac{1}{2} \frac{d^{2}\left\langle\left(\Delta Z_{i}\right)^{2}\right\rangle}{d(\Delta t)^{2}}=0 .
$$

So we expect that the correlation of $u_{i}(t)$ should be small if $\Delta t$ is of the order of the inertial interval; this is not the case for $\mathbf{v}_{i}(t)$.

\section{$3 \quad$ Results}

A pseudo-spectral Direct Numerical Simulation (DNS) code was used to generate homogeneous isotropic incompressible decaying turbulence. Simulations were performed on a periodic cubic grid with each side possessing a length of $2 \pi$ and 64 nodes. A total of 8192 particles were injected into the field at locations with zero scalar (within computational accuracy) with a third-order accurate local cubic interpolation scheme [4] used to obtain the particles' physical velocities and scalar. The fourth-order Adams-Bashforth method was used to move the particles in physical space.

Tables 1 and 2 have details of the properties of the velocity and scalar fields respectively at initialisation of the particles. For the compensated energy spectrum of a high Reynolds number flow, the inertial range should be roughly constant (the Kolmogorov constant); Figure 1, which is the initial spectrum of the simulations for run R35 demonstrates that the field which was used to generate our results possesses this trend. This is a somewhat surprising result 
TABLE 1: Properties (based on the energy spectrum) in sI units of velocity fields at time of injection of particles. $\operatorname{Re}_{\lambda}, t_{\eta}, \eta, \lambda$, $L,\left(\overline{u^{2}}\right)^{1 / 2}, \kappa$ and $\Phi(\kappa)=\varepsilon^{-2 / 3} \kappa^{5 / 3} E(\kappa)$ are the Taylor Reynolds number, Kolmogorov timescale, Kolmogorov, Taylor and integral length scales, RMS velocity, wavenumber and compensated energy spectrum ( $E$ is the turbulent kinetic energy, $\varepsilon$ the turbulent kinetic energy dissipation rate) respectively.

\begin{tabular}{ccccccccc}
\hline Run & $\operatorname{Re}_{\lambda_{0}}$ & $t_{\eta_{0}}$ & $\eta_{0}$ & $\lambda_{0}$ & $L_{0}$ & $\left(\overline{u^{2}}\right)_{0}^{1 / 2}$ & $\kappa_{\max } \eta$ & $\Phi_{\max }$ \\
\hline $\mathrm{R} 20$ & 20.0 & 0.0386 & 0.0684 & 0.602 & 1.13 & 4.03 & 2.01 & 1.85 \\
$\mathrm{R} 25$ & 25.0 & 0.0241 & 0.0541 & 0.532 & 1.04 & 5.70 & 1.59 & 1.96 \\
$\mathrm{R} 30$ & 30.0 & 0.0165 & 0.0448 & 0.483 & 1.01 & 7.55 & 1.32 & 1.92 \\
$\mathrm{R} 35$ & 35.1 & 0.0138 & 0.0410 & 0.478 & 1.00 & 8.92 & 1.21 & 2.08 \\
\hline
\end{tabular}

TABLE 2: Initial properties of scalar fields. $\sigma$ is the Eulerian scalar dispersion, $N$ the scalar dissipation rate.

\begin{tabular}{c|cccc} 
& R20 & R25 & R30 & R35 \\
\hline$\sigma_{0}\left(10^{-4}\right)$ & 2.76 & 6.91 & 15.4 & 24.7 \\
$N_{0}\left(10^{-4} \mathrm{~s}^{-1}\right)$ & 6.44 & 20.2 & 63.4 & 117
\end{tabular}




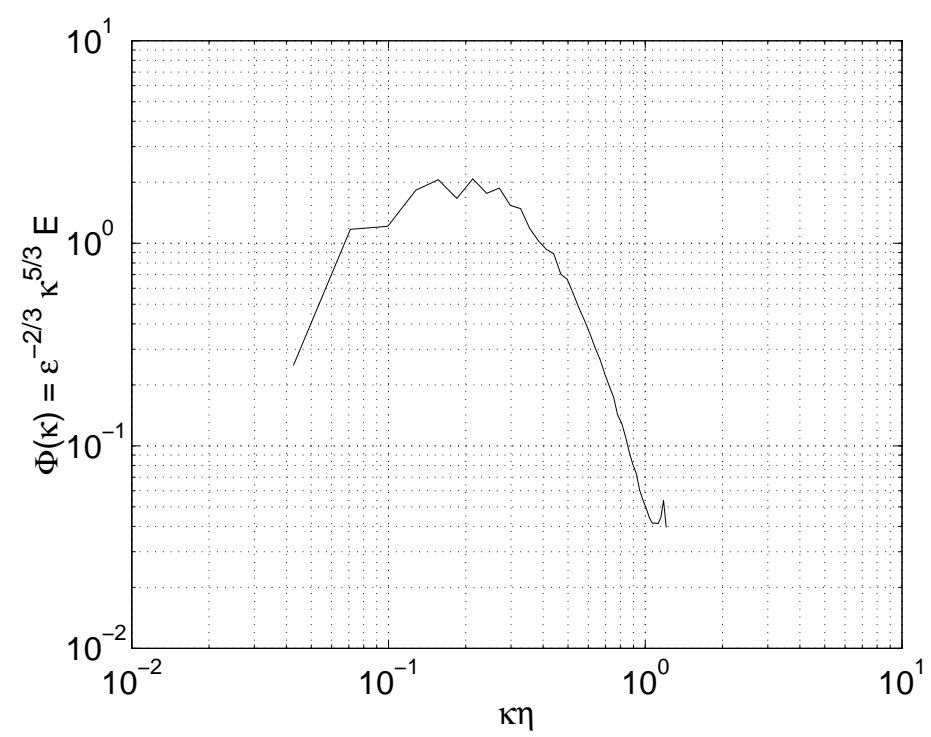

Figure 1: Compensated Velocity Spectrum $\left(\operatorname{Re}_{\lambda}=35\right)$

owing to the relatively low Reynolds number of this field. The Kolmogorov 1941 theory states that the inertial interval exists in the range $1 / L \ll \kappa \ll 1 / \eta$; these limits for our fields were different by only one order of magnitude. The value of the Kolmogorov constant is acceptable [11] - by inspection, it is just below 2. Frisch [2] comments in Section 6.3.2 that nonlinear interactions occur in scales below the Kolmogorov length scale and we note that our fields resolve this region $\left(\kappa_{\max } \eta\right.$ in Table 1$)$; Yeung and Zhou [12] report $\kappa_{\max } \eta \approx 1.5$ for a range of Reynolds numbers. We note that our velocity spectrum suffers from the same effect of aliasing as those reported [12] - the rise in the tail at high wavenumbers - although our spectrum mysteriously drops at the highest resolved wavenumber. Figure 2 is constructed similarly to Figure $8.8[9]$ - we note that the two match quite well. 


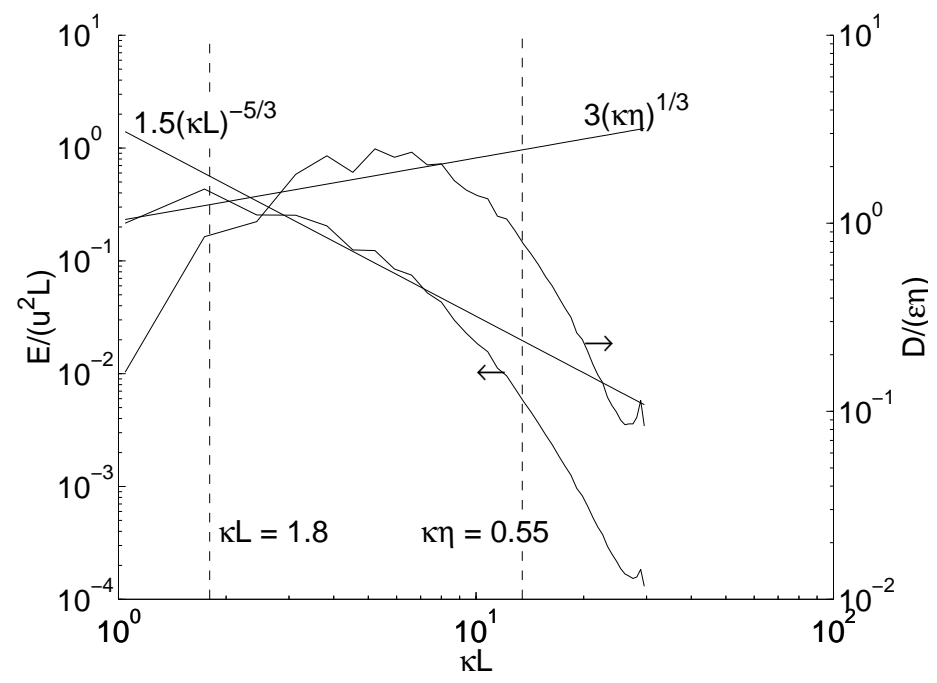

FiguRE 2: Normalised Energy and Dissipation Spectra $\left(\operatorname{Re}_{\lambda}=35\right)$ (cf. Figure 8.8 [9])

Figure 3 contains our results for the decay of the autocorrelations of the scalar and physical velocities. We see that the scalar velocity decays significantly quicker than the physical velocity, as desired. We note that there are some moderate-frequency oscillations in the scalar velocity autocorrelations, which are most pronounced for the highest Reynolds number [Figure 3(d)] and seem to have disappeared by the lowest Reynolds number tested [Figure 3(a)]. These frequencies fall roughly in the range $1 / t_{\eta}<f<2 / t_{\eta}$, which indicates that they are caused by aliasing (recall $\kappa_{\max } \eta$ in Table 1 was in the range 1.2-2). It has been reported [3] that at a Prandtl number of unity (the value used for our simulations), the scalar field requires a higher resolution despite the viscosity and diffusivity being identical. Kerr also reported that aliasing effects were significant for scalar fields with $\operatorname{Pr}=0.7$ at a resolution where the velocity field was not aliased. For our purposes of comparing trends, however, we 


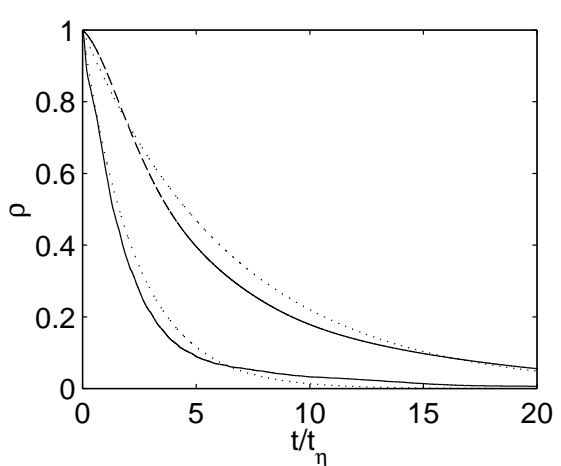

(a)

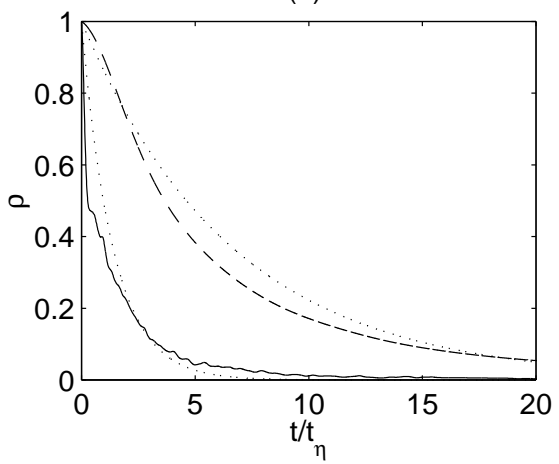

(c)

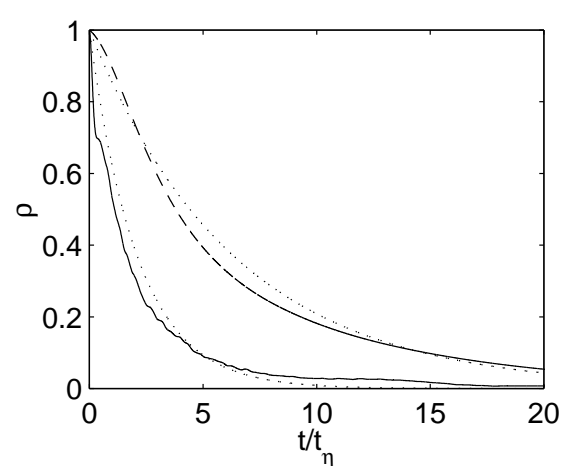

(b)

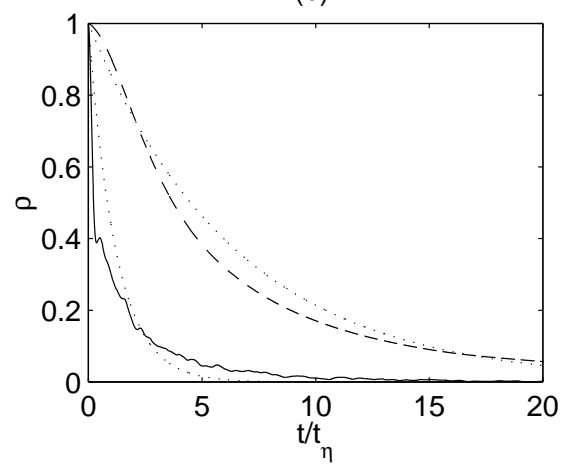

(d)

Figure 3: Time history of velocity autocorrelations. The time axis is normalised by the Kolmogorov timescale. Scalar velocity, - ; physical velocity, - -; exponential approximations [Equation (16)], ... (a) R20; (b) R25; (c) R30; (d) R35. 
see that since the amplitude of these oscillations was relatively low, the overall decay profile was not significantly affected.

\section{Analysis}

Since the Eulerian scalar PDF is Gaussian, the scalar dissipation rate becomes a function only of time $[\langle N \mid Z\rangle \equiv\langle N(t)\rangle]$ and the following simplifications to Equation (5) are possible:

$$
A(Z, t) \equiv-\alpha(t)(Z-\langle Z\rangle) \text { and } B(t) \equiv\langle N(t)\rangle
$$

where

$$
\alpha(t) \equiv 2\langle N(t)\rangle / \sigma(t)
$$

and $\sigma(t)$ is the Eulerian scalar dispersion. In evaluating Equation (7), we used the substitution $\langle u \mid Z\rangle=A(Z, t)$, with $A$ defined by Equation (11). It follows from the PDF equation (2) for homogeneous incompressible turbulence and Gaussian PDF that there is an analytical relationship between the Eulerian dispersion and scalar dissipation rate:

$$
\langle N\rangle=-0.5 d \sigma / d t .
$$

The integral form of this equation was found to have an initial relative error of less than $0.1 \%$ which decayed to a small constant absolute error at large times.

We present in Figure 4 our results of the time history of the Eulerian and Lagrangian scalar dispersions. Also included in this plot is the theoretical Lagrangian dispersion, $\sigma_{L g}$, which can be evaluated by considering Equation (4) and substituting Equation (11) 


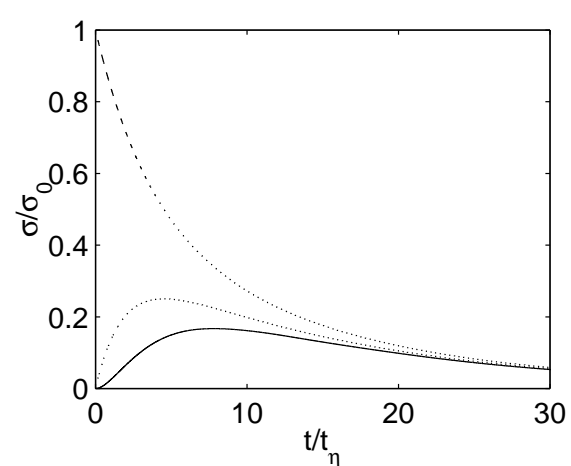

(a)

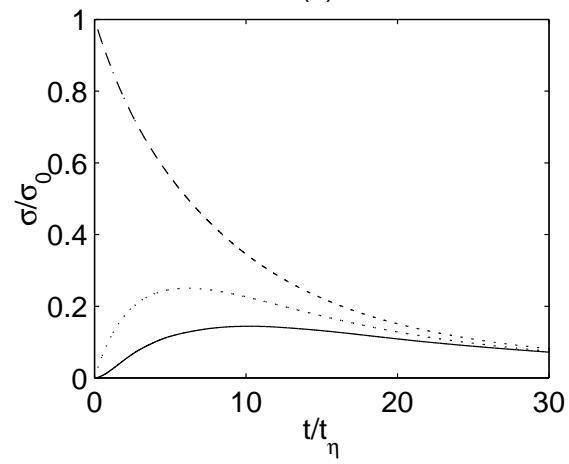

(c)

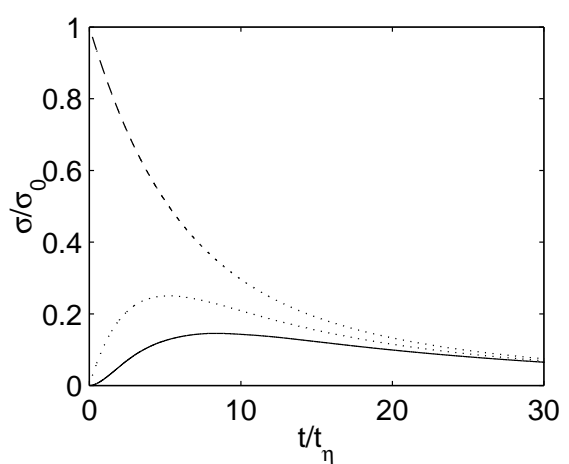

(b)

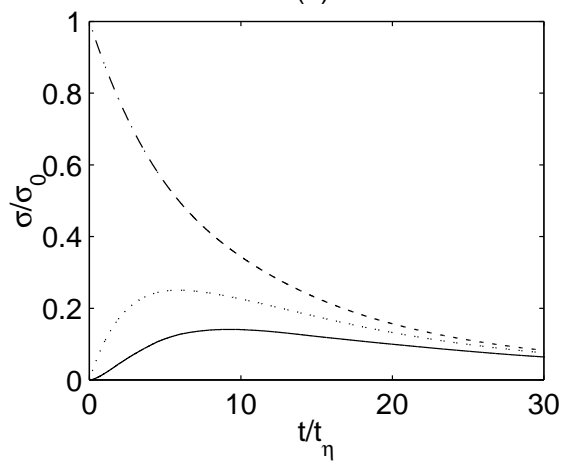

(d)

FiguRE 4: Time history of scalar dispersions normalised by the initial Eulerian scalar dispersion. The time axis is normalised by the Kolmogorov timescale. Lagrangian, —; Eulerian, -.; Theoretical Lagrangian [Equation (14)], ... . (a) R20; (b) R25; (c) R30; (d) R35. 
with the following changes of variable:

$$
\begin{aligned}
\gamma(t) & =-\int_{t_{0}}^{t} \alpha(t) d t, \\
y & =Z \exp (\gamma), \\
f & =F \exp (\gamma), \\
\mathcal{T} & =\int_{t_{0}}^{t} B(t) \exp (2 \gamma) d t .
\end{aligned}
$$

We find that $f$ is a Gaussian with dispersion $2 \mathcal{T}$, hence the dispersion of the Lagrangian PDF, $F$, is:

$$
\sigma_{L g}(t)=g(t) \int_{t_{0}}^{t} \frac{2\langle N\rangle}{g(t)} d t
$$

where

$$
g(t)=\exp \left(-\int_{t_{0}}^{t} \frac{4\langle N\rangle}{\sigma} d t\right) .
$$

Although we see that the computational Lagrangian dispersion never matches the theoretical value until all three converge, this is understandable because the rate of change of Lagrangian dispersion at initialisation is negligible, while the theoretical Lagrangian dispersion's rate is $2\langle N(0)\rangle$.

To compare the autocorrelations for a variety of initial Reynolds numbers, the Lagrangian integral time scales for the autocorrelations [Equations (7) and (8)],

$$
T=\int_{0}^{\infty}|\rho(\Delta t)| d(\Delta t),
$$

were evaluated (the absolute values of the autocorrelations were taken to account for any oscillations about zero). For a stationary Gaussian Markov process [Equation (6)], the autocorrelation is

$$
\rho^{\prime}(\Delta t)=\exp (-|\Delta t| / T),
$$


TABLE 3: Lagrangian integral timescales for scalar and physical velocities

\begin{tabular}{l|llll} 
& R20 & R25 & R30 & R35 \\
\hline$T_{u}$ & 0.0867 & 0.0506 & 0.0226 & 0.0165 \\
$T_{\mathbf{v}}$ & 0.224 & 0.148 & 0.105 & 0.0880
\end{tabular}

which is shown on Figure 3. Although these trends are reasonable approximations, we do not expect them to be exact since we consider here processes which are not necessarily Markovian; we also consider decaying turbulence, which is not stationary.

We present in Table 3 and Figure 5 the data for the various correlation times. See that the correlation time for the scalar process is significantly shorter than the physical process, with the scalar process being comparable to the Kolmogorov scale (as suggested [6]), whereas the physical process is comparable to the integral scale. The scalar velocity correlation times for Reynolds numbers 30 and 35 are probably underestimated owing to the aliasing in scalar data. We also see that Figure 5 compares favourably with results obtained by Wandel, Weinman and Klimenko [10] for a different set of velocity fields, where the Lagrangian physical velocity correlations could not be obtained.

\section{Conclusions}

The theoretical basis of the closure hypothesis of the Conditional Moment Closure (CMC) was that processes in scalar space display similarity with a Markov process. Although aliasing was observed in the scalar results, the amplitude of the aliased frequencies was relatively small. As can be seen in Figure 5, the time taken for a scalar process to become uncorrelated was significantly shorter than 


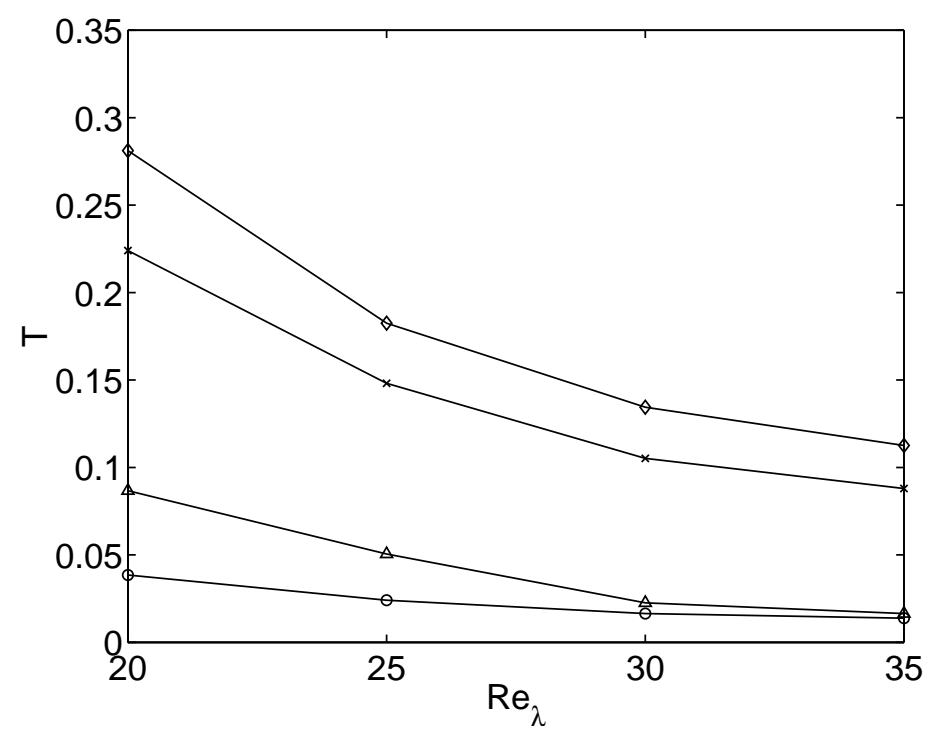

Figure 5: Comparison of correlation times of Lagrangian scalar and physical velocities over a range of initial Taylor Reynolds numbers. Lagrangian scalar velocity [Equation (15)], $\triangle$; Lagrangian physical velocity [Equation (15)], ×; Kolmogorov timescale, o; Integral timescale, $\diamond$. 
the equivalent process in physical space, hence was closer to the characteristics of a Markov process. This validates the derivation of $\mathrm{CMC}$, justifying its application in turbulent reactive flows.

\section{Future work}

We intend to perform simulations at higher Reynolds numbers by using a cubic grid with the number of nodes per side increased to 128 . At this higher resolution, we will also validate the results obtained in this paper. Simulations will be repeated for a Prandtl number of less than unity in an effort to avoid aliasing in the scalar field. We also intend to perform simulations where the turbulence is forced, which would satisfy questions of statistical stationarity (which is inherent in the theoretical analysis) posed by the fact that the fields were decaying.

Acknowledgement: This work has been supported by the Australian Research Council.

\section{References}

[1] R. W. Bilger. Conditional Moment Closure for turbulent reacting flow. Physics of Fluids A, 5:436-444, 1993. C803

[2] U. Frisch. Turbulence: The Legacy of A.N. Kolmogorov. Cambridge University Press, 1995. C809

[3] R. M. Kerr. Higher-order derivative correlations and the alignment of small-scale structures in isotropic numerical 
turbulence. Journal of Fluid Mechanics, 153:31-58, 1985. C810

[4] R. G. Keys. Cubic convolution interpolation for digital image processing. IEEE Transactions on Acoustics, Speech, and Signal Processing, 29(6):1153-1160, 1981. C807

[5] S. H. Kim, K. Y. Huh, and L. Tao. Application of the elliptic Conditional Moment Closure model to a two-dimensional nonpremixed methanol bluff-body flame. Combustion and Flame, 120(1-2):75-90, 2000. C804

[6] A. Y. Klimenko. Multicomponent diffusion of various admixtures in turbulent flow. Fluid Dynamics, 25(3):327-334, 1990. C803, C804, C806, C815

[7] A. Y. Klimenko and R. W. Bilger. Conditional Moment Closure for turbulent combustion. Progress in Energy and Combustion Science, 25(6):595-687, 1999. C803, C804

[8] A. Y. Klimenko, A. P. Wandel, and N. S. A. Smith. Stochastic modelling of scalar transport in turbulent flows based on CMC. Proceedings of ASME International Mechanical Engineering Congress, 1(HTD-24127):1-8, 2001. C805

[9] H. Tennekes and J. L. Lumley. A First Course in Turbulence. MIT Press, Cambridge, Massachusetts, 1994. C809, C810

[10] A. P. Wandel, K. A. Weinman, and A. Y. Klimenko. Diffusion in conserved scalar space: Direct validation by DNS. In B. Moghtaderi, B. Z. D. Dlugogorski, and E. M. Kennedy, editors, 1999 Australian Symposium on Combustion and The Sixth Australian Flame Days, pages 76-80. The Australia and New Zealand Section of the Combustion Institute, The University of Newcastle, 1999. C815 
[11] K. A. Weinman and A. Y. Klimenko. Estimation of the Kolmogorov constant $C_{0}$ by Direct Numerical Simulation of a continuous scalar. Physics of Fluids, 12(12):3205-3220, 2000. C809

[12] P. K. Yeung and Y. Zhou. Universality of the Kolmogorov constant in numerical simulations of turbulence. Physical Review E, 56(2):1746-1752, 1997. C809 